\title{
NGS Reveals Molecular Pathways Affected by Obesity and Weight Loss-Related Changes in miRNA Levels in Adipose Tissue
}

\author{
Alina Kuryłowicz ${ }^{1, *}$ (D), Zofia Wicik ${ }^{1}$, Magdalena Owczarz ${ }^{1}$, Marta Izabela Jonas ${ }^{1}$, \\ Marta Kotlarek ${ }^{2}$, Michał Świerniak ${ }^{2}$, Wojciech Lisik ${ }^{3}$, Maurycy Jonas ${ }^{3}$, \\ Bartłomiej Noszczyk ${ }^{4}$ and Monika Puzianowska-Kuźnicka 1,5,* \\ 1 Department of Human Epigenetics, Mossakowski Medical Research Centre, \\ Polish Academy of Sciences (PAS), 5 Pawinskiego Street, 02106 Warsaw, Poland; \\ zwicik@imdik.pan.pl (Z.W.); mowczarz@imdik.pan.pl (M.O.); martajonas@poczta.onet.pl (M.I.J.) \\ 2 Genomic Medicine, Medical University of Warsaw, 02097 Warsaw, Poland; \\ marta.kotlarek@wum.edu.pl (M.K.); michal.swierniak@wum.edu.pl (M.Ś.) \\ 3 Department of General and Transplantation Surgery, Medical University of Warsaw, 00001 Warsaw, Poland; \\ wojciech.lisik@wum.edu.pl (W.L.); morjon@poczta.onet.pl (M.J.) \\ 4 Department of Plastic Surgery, Medical Centre of Postgraduate Education, 00416 Warsaw, Poland; \\ noszczyk@melilot.pl \\ 5 Department of Geriatrics and Gerontology, Medical Centre of Postgraduate Education, \\ 01826 Warsaw, Poland \\ * Correspondence: akurylowicz@imdik.pan.pl (A.K.); mpuzianowska@imdik.pan.pl (M.P.-K.); \\ Tel.: +48-226-086-406 (A.K.); +48-226-086-591 (M.P.-K.); Fax: +48-226-086-410 (A.K. \& M.P.-K.)
}

Received: 8 December 2017; Accepted: 24 December 2017; Published: 27 December 2017

\begin{abstract}
Both obesity and weight loss may cause molecular changes in adipose tissue. This study aimed to characterize changes in adipose tissue miRNome in order to identify molecular pathways affected by obesity and weight changes. Next generation sequencing (NGS) was applied to identify microRNAs (miRNAs) differentially expressed in 47 samples of visceral (VAT) and subcutaneous (SAT) adipose tissues from normal-weight $(\mathrm{N})$, obese $(\mathrm{O})$ and obese after surgery-induced weight loss (PO) individuals. Subsequently miRNA expression was validated by real-time PCR in 197 adipose tissues and bioinformatics analysis performed to identify molecular pathways affected by obesity-related changes in miRNA expression. NGS identified 344 miRNAs expressed in adipose tissues with $\geq 5$ reads per million. Using $>2$ and $<-2$ fold change as cut-offs we showed that the expression of 54 miRNAs differed significantly between VAT-O and SAT-O. Equally, between SAT-O and SAT-N, the expression of 20 miRNAs differed significantly, between SAT-PO and SAT-N the expression of 79 miRNAs differed significantly, and between SAT-PO and SAT-O, the expression of 61 miRNAs differed significantly. Ontological analyses disclosed several molecular pathways regulated by these miRNAs in adipose tissue. NGS-based miRNome analysis characterized changes of the miRNA profile of adipose tissue, which are associated with changes of weight possibly responsible for a differential regulation of molecular pathways in adipose tissue when the individual is obese and after the individual has lost weight.
\end{abstract}

Keywords: next generation sequencing; miRNome; adipose tissue; obesity; molecular pathways

\section{Introduction}

Studies on the pathogenesis of obesity-related complications point to adipose tissue as the main source of mediators involved in pathological changes of the whole-body function. Transcriptome analyses confirmed substantial aberrations in gene expression in the adipose tissues of obese subjects 
compared to normal-weight individuals [1-4]; however, molecular mechanisms underlying this phenomenon remain largely unknown.

In recent years there has been great progress in understanding the role of microRNA (miRNA) in the regulation of the expression of various genes, including those related to adipocyte differentiation and function. miRNAs implicated in adipogenesis and adipocyte metabolism were found to be differentially expressed in adipose tissue from obese subjects and from normal-weight controls [1,5-8] as well as in different adipose tissue depots [9]. However, these studies were performed with microarrays covering only canonical miRNA sequences deposited in databases. Next generation sequencing (NGS) allows for the identification of novel isoforms of miRNA, and has been successfully used for the analysis of miRNomes in other tissues [10,11].

Precise characterization of miRNome may serve as a basis for identification of cellular functions and pathways disturbed in the course of the investigated pathology [12]. Therefore, we have applied NGS for the identification of miRNAs, the expression of which varies between different adipose tissue depots of obese individuals before and after weight loss, as well as between obese and normal-weight individuals. We have also performed bioinformatics analysis in order to identify gene targets and, subsequently, pathways affected by obesity-related changes in miRNA expression in adipose tissue.

\section{Results}

\section{1. miRNAs Expression in Adipose Tissue}

NGS identified 344 mature miRNAs expressed in adipose tissues of obese, normal-weight and obese-after-weight-loss individuals, for which reads per million (RPM) were $\geq 5$ in at least $50 \%$ of samples within any of the studied groups. Comparison between the analyzed groups revealed significant differences in expression of several miRNAs. Initial analysis showed that the mean expressions of the investigated genes did not differ in adipose tissues of males and females; therefore, all analyses were performed for both sexes together.

miRNAs are deregulated in adipose tissue of obese individuals. Among these miRNAs, the expression of 20 differed between subcutaneous adipose tissue (SAT) samples from obese (O) and normal-weight $(\mathrm{N})$ individuals at the significance level of False Discovery Rate (FDR) $<0.05$, including 19 miRNAs up-regulated $(\mathrm{O} / \mathrm{N}$ fold change $>2$, Table 1 , columns 2 and 3) and 1 down-regulated in SAT-O vs. SAT-N. The most up-regulated miRNAs were hsa-miR-146b-3p, hsa-miR-146b-5p, hsa-miR-223-3p, hsa-miR-450b-5p and hsa-miR-22-3p, while hsa-miR-205-5p was deeply down-regulated.

Table 1. Fold changes of microRNAs (miRNAs) differentially expressed in subcutaneous adipose tissue (SAT) of obese individuals before (O) and after weight loss (PO), as well as of normal-weight subjects (N) assessed by the next generation sequencing (NGS) and real-time PCR methods.

\begin{tabular}{|c|c|c|c|c|c|c|}
\hline & \multicolumn{2}{|c|}{ SAT-O vs. SAT-N } & \multicolumn{2}{|c|}{ SAT-PO vs. SAT-O } & \multicolumn{2}{|c|}{ SAT-PO vs. SAT-N } \\
\hline & NGS & Real-Time PCR & NGS & Real-Time PCR & NGS & Real-Time PCR \\
\hline 1 & 2 & 3 & 4 & 5 & 6 & 7 \\
\hline \multicolumn{7}{|l|}{ Proadipogenic } \\
\hline hsa-miR-15a-5p * & & & -2.992 & & -2.286 & \\
\hline hsa-miR-21-5p * & 2.728 & & & & & \\
\hline hsa-miR-107 & & & -3.057 & & -3.734 & \\
\hline hsa-miR-143-5p * & & & -2.126 & & & \\
\hline hsa-miR-146b-3p & 7.253 & 2.710 & -4.722 & -2.972 & & \\
\hline hsa-miR-150-5p * & & & & & 2.128 & \\
\hline hsa-miR-194-5p & & & -3.029 & -3.645 & -2.109 & -7.00 \\
\hline hsa-miR-199b-5p & & & & & 2.803 & \\
\hline hsa-miR-210 & & & -2.130 & & -2.617 & \\
\hline hsa-miR-214-5p * & & & & & 2.266 & \\
\hline hsa-miR-335-3p & & & & & 2.574 & \\
\hline hsa-miR-450a-5p & 2.282 & & & & 2.100 & \\
\hline
\end{tabular}


Table 1. Cont

\begin{tabular}{|c|c|c|c|c|c|c|}
\hline & \multicolumn{2}{|c|}{ SAT-O vs. SAT-N } & \multicolumn{2}{|c|}{ SAT-PO vs. SAT-O } & \multicolumn{2}{|c|}{ SAT-PO vs. SAT-N } \\
\hline & NGS & Real-Time PCR & NGS & Real-Time PCR & NGS & Real-Time PCR \\
\hline 1 & 2 & 3 & 4 & 5 & 6 & 7 \\
\hline \multicolumn{7}{|l|}{ Antiadipogenic } \\
\hline hsa-miR-18a-3p & & & -3.703 & & -3.699 & \\
\hline hsa-miR-23a-5p & 2.093 & & & & & \\
\hline hsa-miR-27a-5p * & 2.363 & & & & 2.512 & \\
\hline hsa-miR-29a-3p * & & & & & 2.010 & \\
\hline hsa-miR-130b-3p & & & -4.053 & & -2.787 & \\
\hline hsa-miR-196a-5p & & & 4.031 & 3.162 & 4.047 & 2.97 \\
\hline \multicolumn{7}{|c|}{ Involved in adipocyte browning } \\
\hline hsa-miR-125b-5p & & & & & 2.247 & 2.01 \\
\hline \multicolumn{7}{|c|}{ Involved in inflammation and immune response } \\
\hline hsa-miR-15b-3p * & & & -5.090 & & -6.432 & \\
\hline hsa-miR-16-2-3p * & & & -7.972 & & -6.263 & \\
\hline hsa-miR-20b-5p * & & & -2.936 & & -3.518 & \\
\hline hsa-miR-22-3p * & 3.001 & 2.011 & & & & \\
\hline hsa-miR-32-5p * & & & -2.452 & & & \\
\hline hsa-miR-92b-3p * & & & & & 2.228 & \\
\hline hsa-miR-93-5p & & & -3.602 & & -3.358 & \\
\hline hsa-miR-106a-5p * & & & -3.470 & & -4.302 & \\
\hline hsa-miR-122-5p * & & & -5.792 & & & \\
\hline hsa-miR-125a-5p * & & & & & 2.150 & 1.96 \\
\hline hsa-miR-125b-1-3p * & 2.063 & & & & & \\
\hline hsa-miR-142-3p * & & & -5.215 & & & \\
\hline hsa-miR-142-5p * & & & -6.087 & & -5.233 & \\
\hline hsa-miR-146b-5p * & 5.077 & & -3.986 & & & \\
\hline hsa-miR-182-5p * & & & -15.039 & & & \\
\hline hsa-miR-223-3p * & 5.804 & 3.041 & -27.825 & -8.036 & -4.794 & -2.72 \\
\hline hsa-miR-3200-3p & & & -3.103 & & -3.802 & \\
\hline \multicolumn{7}{|c|}{ Involved in lipid metabolism } \\
\hline hsa-miR-106b-5p * & & & -3.778 & & -3.984 & \\
\hline hsa-miR-144-3p * & & & -11.390 & & -14.187 & \\
\hline hsa-miR-185-5p & & & -2.836 & & -3.303 & \\
\hline \multicolumn{7}{|c|}{ Involved in atherosclerosis } \\
\hline hsa-miR-365a-3p & & & & & 2.559 & \\
\hline hsa-miR-365b-3p & & & & & 2.559 & \\
\hline \multicolumn{7}{|c|}{ Involved in insulin signaling } \\
\hline hsa-miR-96-5p & & & -22.917 & -9.251 & -13.913 & -15.01 \\
\hline hsa-miR-128 & & & -2.418 & & & \\
\hline \multicolumn{7}{|c|}{ Involved in oncogenesis } \\
\hline hsa-let-7e-3p & & & & & 2.100 & \\
\hline hsa-miR-16-5p & & & -2.755 & & & \\
\hline hsa-miR-17-3p & & & -2.066 & & & \\
\hline hsa-miR-18a-5p & & & -2.410 & & -4.324 & \\
\hline hsa-miR-18b-5p & & & -4.240 & & -6.464 & \\
\hline hsa-miR-25-3p & & & -2.289 & & -2.448 & \\
\hline hsa-miR-28-5p & & & & & 2.086 & \\
\hline hsa-miR-95 & & & & & 3.118 & \\
\hline hsa-miR-106b-3p & & & -3.636 & & & \\
\hline hsa-miR-144-5p & & & -8.083 & & -8.337 & \\
\hline hsa-miR-183-5p & & & -14.823 & -3.723 & -20.421 & -7.62 \\
\hline hsa-miR-192-5p & & & -3.234 & & -3.408 & \\
\hline hsa-miR-193b-5p & & & & & 2.500 & 1.97 \\
\hline hsa-miR-205-5p & -10.897 & -5.541 & & & -10.030 & -10.73 \\
\hline
\end{tabular}


Table 1. Cont

\begin{tabular}{|c|c|c|c|c|c|c|}
\hline & \multicolumn{2}{|c|}{ SAT-O vs. SAT-N } & \multicolumn{2}{|c|}{ SAT-PO vs. SAT-O } & \multicolumn{2}{|c|}{ SAT-PO vs. SAT-N } \\
\hline & NGS & Real-Time PCR & NGS & Real-Time PCR & NGS & Real-Time PCR \\
\hline 1 & 2 & 3 & 4 & 5 & 6 & 7 \\
\hline \multicolumn{7}{|c|}{ Involved in oncogenesis } \\
\hline hsa-miR-215 & 2.015 & & & & & \\
\hline hsa-miR-223-5p & 2.894 & & -5.798 & & -2.003 & \\
\hline hsa-miR-301b & 2.972 & & & & & \\
\hline hsa-miR-330-3p & & & & & 2.452 & \\
\hline hsa-miR-340-3p & & & & & 2.073 & \\
\hline hsa-miR-361-3p & & & & & 2.504 & \\
\hline hsa-miR-363-3p & & & -5.212 & & -4.293 & \\
\hline hsa-miR-423-5p & & & -2.430 & & & \\
\hline hsa-miR-424-3p & & & -2.020 & & & \\
\hline hsa-miR-425-5p & & & -3.062 & & -2.103 & \\
\hline hsa-miR-451a & & & -9.299 & & -11.488 & \\
\hline hsa-miR-483-3p & & & 2.681 & & 2.860 & \\
\hline hsa-miR-489 & & & & & 2.440 & \\
\hline hsa-miR-501-3p & & & -3.074 & & & \\
\hline hsa-miR-518b & 2.705 & & & & & \\
\hline hsa-miR-548d-5p & & & -3.669 & & -3.766 & \\
\hline hsa-miR-550a-3p & & & -3.100 & & & \\
\hline hsa-miR-576-5p & & & -3.083 & & -3.587 & \\
\hline hsa-miR-584-5p & & & -2.741 & & -2.377 & \\
\hline hsa-miR-615-3p & & & & & 3.163 & \\
\hline hsa-miR-628-5p & 2.569 & & & & 2.504 & \\
\hline hsa-miR-629-5p & & & -2.241 & & -2.261 & \\
\hline hsa-miR-652-3p & & & -2.807 & & -2.653 & \\
\hline hsa-miR-671-3p & & & & & 2.878 & \\
\hline hsa-miR-874 & & & & & 2.070 & \\
\hline hsa-miR-891a & 2.984 & & & & & \\
\hline hsa-miR-941 & 2.404 & & -2.239 & & & \\
\hline hsa-miR-1260b & & & 2.460 & 1.524 & 4.854 & 2.30 \\
\hline hsa-miR-1271-5p & 2.540 & & & & & \\
\hline hsa-miR-1285-3p & & & -2.396 & & & \\
\hline hsa-miR-3688-3p & & & -5.070 & & -5.732 & \\
\hline hsa-miR-4662a-5p & & & & & 3.410 & \\
\hline hsa-miR-4732-3p & & & -3.347 & & -3.124 & \\
\hline hsa-miR-5683 & & & & & 5.557 & \\
\hline hsa-miR-6716-3p & & & & & 2.481 & \\
\hline \multicolumn{7}{|l|}{ Involved in ageing } \\
\hline hsa-miR-141-3p & & & -5.518 & & -8.255 & \\
\hline hsa-miR-369-5p & & & & & 2.554 & \\
\hline \multicolumn{7}{|c|}{ Involved in cardiovascular diseases } \\
\hline hsa-miR-589-5p & & & & & 2.193 & \\
\hline hsa-miR-3615 & & & -5.278 & & -4.355 & \\
\hline \multicolumn{7}{|c|}{ Involved in neurodegenerative disorders } \\
\hline hsa-miR-15b-5p & & & -2.811 & & -2.753 & \\
\hline hsa-miR-132-5p & 2.921 & & & & & \\
\hline hsa-miR-151a-5p & & & & & 2.228 & \\
\hline hsa-miR-548ay-5p & & & -3.505 & & -3.731 & \\
\hline hsa-miR-664a-3p & & & & & 2.102 & \\
\hline hsa-miR-3607-3p & & & & & 2.706 & \\
\hline hsa-miR-3909 & & & & & 2.865 & \\
\hline \multicolumn{7}{|l|}{ Unknown function } \\
\hline hsa-miR-450b-5p & 3.284 & 3.723 & & & 2.605 & 7.13 \\
\hline hsa-miR-486-3p & & & -12.206 & & -11.022 & \\
\hline hsa-miR-486-5p & & & -9.535 & -4.464 & -9.118 & -8.83 \\
\hline
\end{tabular}

* miRNA also associated with other obesity-related pathologies. 
Furthermore, the expression of only one miRNA, hsa-miR-424-3p, was significantly up-regulated in visceral adipose tissue of obese subjects (VAT-O) compared to VAT-N (O/N fold change = 2.63 in NGS). None of the miRNAs down-regulated in VAT-O vs. VAT-N passed the cut-off criteria of statistical significance (FDR < 0.05).

Obesity was associated with a significantly higher expression of 47 miRNAs including the most up-regulated hsa-miR-31-5p, hsa-miR-200a-3p, hsa-miR-200b-3p, hsa-miR-204-5p, hsa-miR-429 and hsa-miR-493-3p; it was also associated with a lower expression of seven miRNAs: hsa-miR-23a-5p, hsa-miR-27a-5p, hsa-miR-96-5p, hsa-miR-183-5p, hsa-miR-196a-5p, hsa-miR-486-5p and hsa-miR-615-3p in VAT-O compared to SAT-O (Table 2). In contrast, no significant differences in miRNAs expression between VAT-N and SAT-N were observed in normal-weight individuals.

Table 2. Fold changes of miRNA differentially expressed in visceral (VAT) and subcutaneous adipose tissue (SAT) of obese $(\mathrm{O})$ individuals assessed by the next generation sequencing (NGS) and the real-time PCR methods.

\begin{tabular}{|c|c|c|}
\hline & \multicolumn{2}{|c|}{ VAT-O vs. SAT-O } \\
\hline & NGS & Real-Time PCR \\
\hline \multicolumn{3}{|l|}{ Proadipogenic } \\
\hline hsa-miR-450a-5p & 2.023 & \\
\hline \multicolumn{3}{|l|}{ Antiadipogenic } \\
\hline hsa-miR-23a-5p & -3.371 & \\
\hline hsa-miR-27a-5p & -2.512 & \\
\hline hsa-miR-29b-3p & 2.471 & \\
\hline hsa-miR-29c-3p & 2.084 & \\
\hline hsa-miR-196a-5p & -14.684 & -17.21 \\
\hline \multicolumn{3}{|c|}{ Involved in adipogenesis } \\
\hline hsa-miR-125b-5p & 2.084 & 1.89 \\
\hline \multicolumn{3}{|c|}{ Involved in adipocytes browning } \\
\hline hsa-miR-539-3p & 2.127 & \\
\hline \multicolumn{3}{|c|}{ Involved in inflammation and immune response } \\
\hline hsa-miR-125a-5p & 2.615 & 1.98 \\
\hline \multicolumn{3}{|l|}{ Insulin signaling } \\
\hline hsa-miR-33a-3p & 2.283 & \\
\hline hsa-miR-33b-5p & 3.170 & \\
\hline hsa-miR-96-5p & -2.887 & -2.23 \\
\hline hsa-miR-133a & 2.720 & \\
\hline hsa-miR-133b & 2.665 & \\
\hline \multicolumn{3}{|c|}{ Involved in oncogenesis } \\
\hline hsa-let-7e-3p & 2.145 & \\
\hline hsa-miR-31-5p & 10.078 & \\
\hline hsa-miR-100-3p & 2.979 & \\
\hline hsa-miR-100-5p & 2.647 & \\
\hline hsa-miR-101-5p & 2.647 & \\
\hline hsa-miR-136-5p & 2.562 & \\
\hline hsa-miR-183-5p & -2.780 & -1.98 \\
\hline hsa-miR-190a & 2.017 & \\
\hline hsa-miR-193a-3p & 3.319 & 2.03 \\
\hline hsa-miR-200a-3p & 5.333 & \\
\hline hsa-miR-200b-3p & 5.784 & \\
\hline hsa-miR-203a & 4.713 & \\
\hline hsa-miR-204-5p & 6.024 & \\
\hline hsa-miR-218-5p & 2.537 & \\
\hline hsa-miR-337-3p & 2.051 & \\
\hline hsa-miR-376c-3p & 2.908 & \\
\hline hsa-miR-381-3p & 2.405 & \\
\hline hsa-miR-424-5p & 2.573 & \\
\hline
\end{tabular}


Table 2. Cont

\begin{tabular}{lcc}
\hline & \multicolumn{2}{c}{ VAT-O vs. SAT-O } \\
\hline & NGS & Real-Time PCR \\
\hline Involved in oncogenesis & & \\
\hline hsa-miR-429 & 5.626 & -2.67 \\
hsa-miR-486-5p & -2.872 & \\
hsa-miR-487b & 2.002 & \\
hsa-miR-493-3p & 5.581 & \\
hsa-miR-493-5p & 5.259 & \\
hsa-miR-497-5p & 2.083 \\
hsa-miR-532-3p & 2.006 \\
hsa-miR-551b-3p & 4.019 \\
hsa-miR-561-5p & 2.275 \\
hsa-miR-615-3p & -3.246 \\
hsa-miR-887 & 2.400 \\
hsa-miR-1307-5p & 2.601 \\
\hline Involved in ageing & \\
\hline hsa-miR-299-3p & 3.240 \\
\hline Involved in cardiovascular disorders & \\
\hline hsa-miR-423-3p & 2.013 \\
hsa-miR-495-3p & 2.702 \\
\hline Involved in neurodegenerative disorders \\
\hline hsa-miR-9-5p & 2.536 \\
hsa-miR-101-3p & 2.979 \\
hsa-miR-136-3p & 2.436 \\
hsa-miR-376a-5p & 2.099 \\
hsa-miR-382-3p & 2.184 \\
hsa-miR-485-3p & 2.122 \\
hsa-miR-3607-3p & 3.016 \\
\hline
\end{tabular}

\subsection{Weight Loss Is Associated with Change of the SAT miRNA Profile}

Next, we analyzed changes in the miRNA profile associated with weight loss. Expression of 61 miRNAs differed significantly between SAT samples obtained from obese subjects before $(\mathrm{O})$ and after weight loss (PO) with a fold change at least $>2$ or $<-2$ (Table 1, columns 4 and 5). The expression of three miRNAs: hsa-miR-196a-5p, hsa-miR-483-3p and has-miR-1260b was higher in SAT-PO compared to SAT-O, and the expression of 58 miRNAs, including hsa-miR-96-5p, hsa-miR-144-3p, hsa-miR-182-5p, hsa-miR-183-5p, hsa-miR-223-3p and hsa-miR-486-3p, was lower in SAT-PO than in SAT-O.

A subgroup of miRNAs, namely hsa-miR-146b-3p, hsa-miR-146b-5p, hsa-miR-223-3p, hsa-miR-223-5p and hsa-miR-941, the expression of which was significantly decreased after weight loss, were also identified as up-regulated in SAT-O compared to SAT-N.

\section{3. miRNA Profile in Adipose Tissue after Weight Loss Differs from That in Adipose Tissue of Normal-Weight Individuals}

Finally, we addressed the question as to whether miRNA expression profile differed between SAT samples obtained from the obese subjects after weight loss compared to normal-weight individuals. We found that the level of 79 miRNAs was significantly different, with a fold change at least $>2$ or $<-2$ (Table 1, columns 6 and 7). At the top of the list of the 42 miRNAs with lower expression in SAT-PO than in SAT-N were hsa-miR-96-5p, hsa-miR-144-3p, hsa-miR-183-5p, hsa-miR-205-5p, hsa-miR-451a, hsa-miR-486-3p and -5p, while hsa-miR-95, hsa-miR-196a-5p, hsa-miR-615-3p and hsa-miR-1260b headed the list of 37 miRNA with higher expression in SAT-PO than in SAT-N.

Three miRNAs, hsa-miR-27a-5p, hsa-miR-450b-5p and hsa-miR-628-5p, with a higher expression in SAT-O than in SAT-N, remained increased in SAT-PO, while the expression of hsa-miR-205-5p remained reduced. Out of five miRNAs whose expression was significantly increased in SAT-O 
compared to SAT-N and subsequently decreased after weight loss (hsa-miR-146b-3p, hsa-miR-146b-5p, hsa-miR-223-3p, hsa-miR-223-5p and hsa-miR-941) only hsa-miR-223-3p and -5p had their levels after weight loss significantly lower than in normal-weight subjects.

\subsection{Real Time PCR Verification of the NGS Results}

Real-time PCR (RT-PCR) positively confirmed the obesity-associated expression changes of the selected miRNAs detected by NGS (Tables 1 and 2). On the other hand, out of three miRNAs with the most stable expression in NGS, only hsa-miR-374a-5p had comparable levels in adipose tissues regardless of weight and weight changes when assessed by real-time PCR, while hsa-miR-214-3p and hsa-miR-342-3p levels were significantly higher in SAT-PO than in SAT-O, indicating that they cannot be used as internal control for miRNA testing in adipose tissue.

\subsection{Identification of Target Genes Regulated by Differentially Expressed miRNAs in Adipose Tissues}

Using the MirWalk and MirTarBase programs, we searched for genes regulated by miRNAs whose expression in adipose tissue was significantly (fold change at least $>2$ or $<-2$ ) affected by obesity and weight loss. We identified 9932 putative gene targets for miRNAs differentially expressed in SAT-O and SAT-N, 16158 targets for miRNAs differentially expressed in SAT-PO and SAT-O, 17186 targets of miRNAs whose levels differed significantly between SAT-PO and SAT-N, and 15565 targets regulated by miRNAs differentially expressed in VAT-O and SAT-O. Analysis of targets was performed separately for each abovementioned pair of tissues. Notably, targets with a known function in obesity (Table 3) were among those with the highest $(>10)$ number of binding sites for miRNAs characterized by differential expression.

Table 3. Common obesity-related targets with the highest $(>10)$ number of binding sites for miRNAs differentially expressed in subcutaneous (SAT) and visceral (VAT) adipose tissues of normal weight (N) and obese before (O) and after surgery (PO) individuals.

\begin{tabular}{|c|c|c|c|}
\hline SAT-O vs. SAT-N & SAT-O vs. SAT-PO & SAT-N vs. SAT-PO & VAT-O vs. SAT-O \\
\hline PPARA & PRKAR1A & $V E G F A$ & $A D R B 2$ \\
\hline CASP9 & CCND1 & CREB1 & VEGFA \\
\hline PPARGC1B & PTEN & $B C L 2$ & BCL2 \\
\hline IGF1R & CDKN1A & FLT1 & SCARB1 \\
\hline FMR1 & $H M G A 2$ & MDM2 & CDC42 \\
\hline$S L C 26 A 2$ & HMGA1 & CCR2 & PAX 8 \\
\hline$R A D 51 B$ & XIAP & PCDH15 & SP1 \\
\hline MBD4 & CCND2 & EXOC7 & SLC16A1 \\
\hline FOXP1 & SMAD4 & $A R L 17 A$ & BICD1 \\
\hline RNASEL & CADM1 & PARD3 & CSNK1D \\
\hline CAMTA1 & SPRED1 & BICD1 & MCL1 \\
\hline BMPR2 & MBD1 & $A D R A 1 A$ & IL1RAP \\
\hline VIPR2 & HSPA1B & TMPO & CLTC \\
\hline$D B T$ & NOTCH2 & QKI & CELF2 \\
\hline CDS1 & BRWD1 & DNAJC10 & ENAH \\
\hline WHSC1L1 & E2F3 & ZNF496 & HSPA13 \\
\hline CELF1 & MAPK9 & & PCDH7 \\
\hline DAPK2 & NRG1 & & HDGF \\
\hline PHF20 & $S L C 2 A 3$ & & DYNC1I1 \\
\hline CRYL1 & KIF6 & & PPP1R12B \\
\hline CCDC13 & HMBOX1 & & \\
\hline & TPGS2 & & \\
\hline
\end{tabular}

Next, we used our estimation method described in the Methods section to evaluate the direction of the expression changes of 10 putative targets that might have been affected by the obesity-related changes in miRNA levels. For this analysis we selected BMPR2 (encoding bone morphogenic protein receptor 2), CABP4 (encoding calcium binding protein 4), CFL2 (encoding cofilin 2), DBT (encoding lipoamide acyltransferase component of branched-chain alpha-keto acid dehydrogenase complex), 
DISC1 (encoding disrupted in schizophrenia 1), DOK1 (encoding docking protein 1), FOXP1 (encoding forkhead box protein P1), IGF1R (encoding insulin-like growth factor receptor 1), MTMR12 (encoding myotubularin related protein 12) and TRIM14 (encoding tripartite motif-containing protein 14) whose expression, based on the miRNA analysis, should be affected in all analyzed pairs of tissues. In order to establish the relevance of the above analysis, we measured the expression of these targets by real-time PCR. We found that in each case the direction of expression changes predicted by our estimation method agreed with the experimental data (Table S1).

Apart from obesity-related genes (Figure 1a), among potential targets for miRNAs differentially expressed in the investigated tissues were also those associated with obesity-related complications including diabetes (Figure 1b), oxidative stress (Figure 1c) and atherosclerosis (Figure 1d).

a.

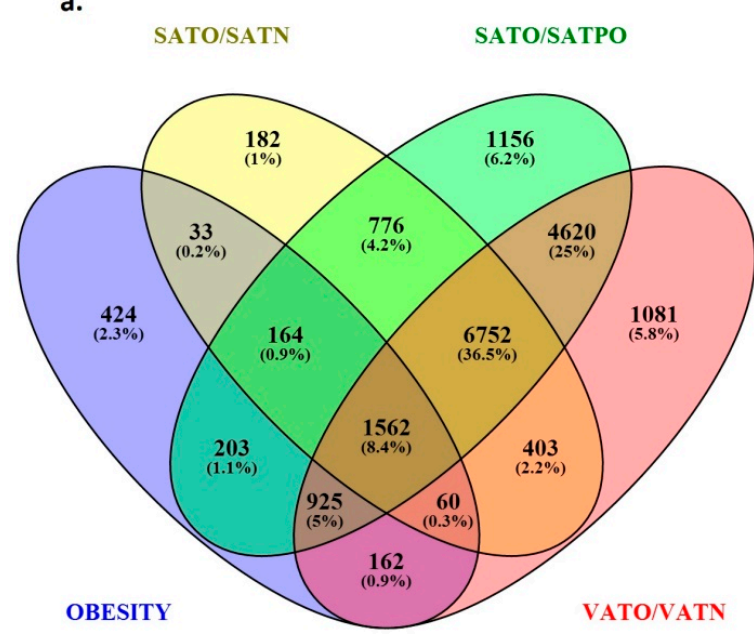

c.

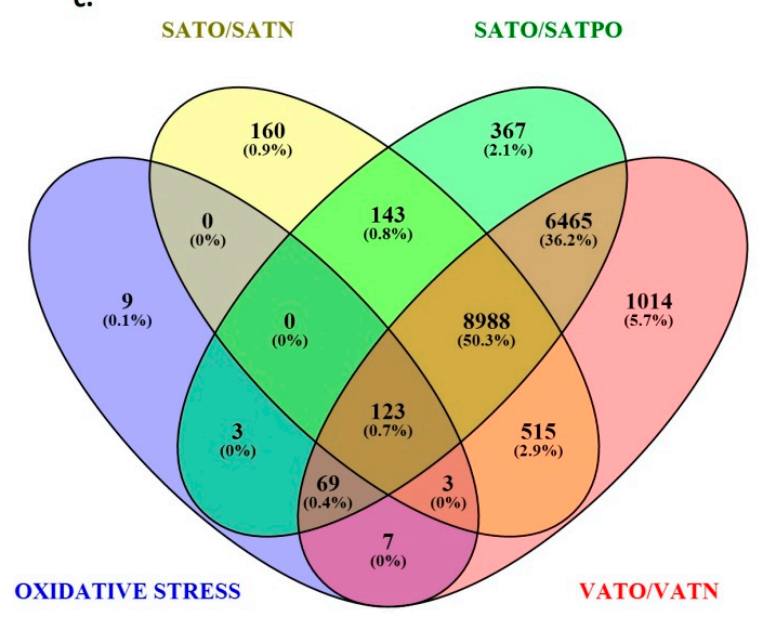

b.

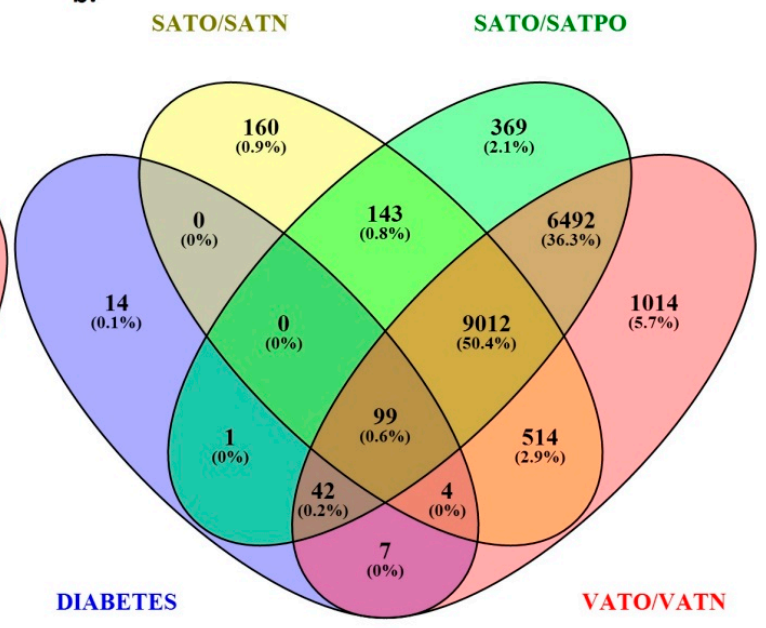

d.

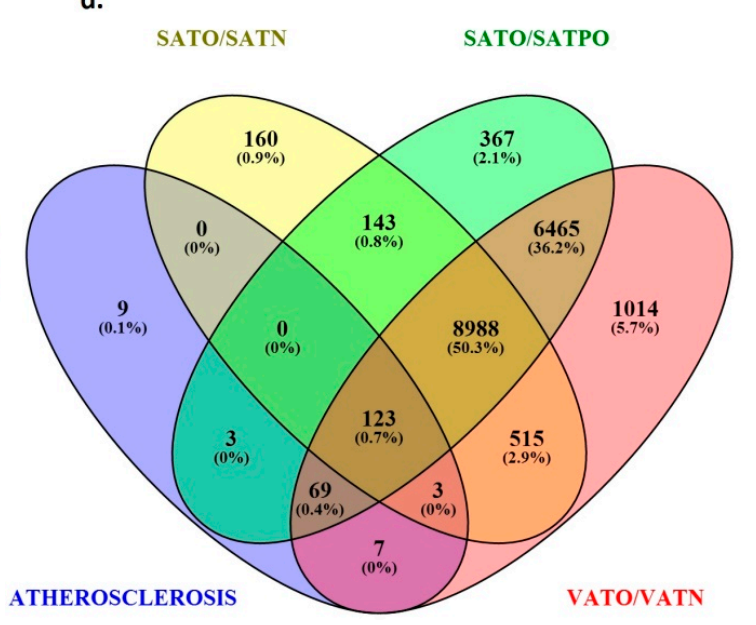

Figure 1. Venn's diagrams showing involvement of genes regulated by miRNAs differentially expressed in subcutaneous (SAT) and visceral (VAT) adipose tissues from obese (O), normal weight (N), obese after weight-loss (PO) individuals in pathogenesis of obesity (a), diabetes (b), oxidative stress (c) and atherosclerosis $(\mathbf{d})$.

\subsection{Molecular Pathways Regulated by Differentially Expressed miRNAs}

To identify the molecular pathways associated with a change in the obesity-related miRNome, we employed the PANTHER Classification System. When potentially affected targets were taken into account, 49 pathways were indicated (a summary of the most relevant results is presented in Table 4), 
some of them overrepresented, some underrepresented, suggesting that obesity is associated with either greater or lower than expected, respectively, regulation of a given pathway by these miRNAs. Among the pathways, only three passed the Bonferroni correction (Table 4).

Table 4. Selected molecular pathways significantly regulated by differentially expressed miRNAs in subcutaneous (SAT) and visceral (VAT) adipose tissues of normal-weight $(\mathrm{N})$, obese $(\mathrm{O})$ and after weight loss (PO) individuals. Values presented as fold enrichment (values $>1$ correspond to the overrepresentation of the particular pathway while values $<1$ to its underrepresentation).

\begin{tabular}{|c|c|c|c|c|}
\hline & \multicolumn{4}{|c|}{ Fold Enrichment } \\
\hline & $\begin{array}{l}\text { SAT-O vs. } \\
\text { SAT-N }\end{array}$ & $\begin{array}{l}\text { SAT-PO vs. } \\
\text { SAT-O }\end{array}$ & $\begin{array}{l}\text { SAT-PO vs. } \\
\text { SAT-N }\end{array}$ & $\begin{array}{l}\text { VAT-O vs. } \\
\text { SAT-O }\end{array}$ \\
\hline \multicolumn{5}{|l|}{ Pathways corrected * } \\
\hline $\begin{array}{l}\text { Inflammation mediated by chemokine and cytokine } \\
\text { signaling pathway (P00031) }\end{array}$ & 1.22 & -1.13 & -1.14 & 1.17 \\
\hline Wnt signaling pathway (P00057) & & & & -1.53 \\
\hline CCKR signaling map (P06959) & & & 1.35 & \\
\hline \multicolumn{5}{|l|}{ Pathways non corrected ** } \\
\hline Beta2 adrenergic receptor signaling pathway (P04378) & -2.46 & & & \\
\hline Interleukin signaling pathway (P00036) & & & & 1.23 \\
\hline Endothelin signaling pathway (P00019) & 1.46 & & & \\
\hline Apoptosis signaling pathway (P00006) & 1.25 & 1.81 & -1.23 & -1.47 \\
\hline p53 pathway (P00059) & 1.30 & 1.78 & -1.28 & -1.68 \\
\hline p53 pathway by glucose deprivation (P04397) & 1.63 & & & \\
\hline Alzheimer disease-presenilin pathway (P00004) & -1.28 & & & -1.47 \\
\hline Glycolysis (P00024) & & 3.55 & & -2.64 \\
\hline Cholesterol biosynthesis (P00014) & & & & -2.44 \\
\hline
\end{tabular}

* Pathways that passed the Bonferroni correction; ** Pathways that did not pass the Bonferroni correction.

\section{Discussion}

In this work, we present the results of a comprehensive analysis of adipose tissue miRNome changes associated with excess adiposity. We showed that some obesity-related changes in adipose tissue miRNome do not disappear after weight loss. Consequently, based on in silico analysis, we identified molecular pathways differentially regulated in adipose tissue from normal-weight and obese subjects before and after weight loss.

Among the miRNAs up-regulated in the SAT of obese subjects compared to the SAT of normal-weight controls were those with proadipogenic (e.g., hsa-miR-21-5p, hsa-miR-146b-3p and hsa-miR-450a-5p) as well as those with antiadipogenic properties (hsa-miR-23a-5p and hsa-miR-27a-5p), supporting the notion that obesity is associated with dysregulation of adipogenesis [13]. Apart from hsa-miR-21 and hsa-miR-146b, none of these miRNAs has been previously reported to be up-regulated in SAT-O [12,14,15]. Notably, hsa-miR-450 was found to be down-regulated in adipocyte cell lines isolated from obese patients [7]. This discrepancy could have resulted from the fact that the microarray technique used by Ortega et al. did not distinguish hsa-miR-450 isoforms. Other miRNAs up-regulated in SAT-O were those involved in the regulation of the immune response, acting as suppressors (e.g., hsa-miR-223-3p) or activators (e.g., hsa-miR-146b-5p) of inflammation $[12,16,17]$. Ontological analysis pointed to an overrepresentation of inflammatory pathways mediated by chemokine and cytokine signaling in SAT-O as compared to SAT-N (Table 4). "Overrepresentation" refers to the increased regulation of a given pathway, but does not firmly indicate whether it is up- or down-regulated; this might only be estimated from the level of expression of miRNAs regulating a given pathway. Nevertheless, literature data point to an increased proinflammatory activity of adipose tissue in obese individuals $[18,19]$. Other molecular pathways overrepresented in adipose tissue in obesity were those associated with endothelin signaling, apoptosis and p53 action. Experimental data suggest that obesity is associated with an increased endothelin 
concentration and the pro-apoptotic phenotype of adipose tissue [20-22]. In obesity, the beta-2 adrenergic receptor signaling pathway was found to be underrepresented in SAT, and our previous findings point to the lower expression of genes encoding adrenergic beta receptors in adipose tissues of obese individuals compared to tissues obtained from normal-weight subjects [23]. We found hsa-miR-205-5p to be the only gene deeply down-regulated in SAT-O compared to SAT-N. This miRNA has not been previously reported as associated with obesity, however among its targets are those involved in the pathogenesis of obesity-related complications, e.g., genes encoding interleukins (IL-6, $I L-17 B$ and $I L-22)$, apolipoproteins (APOA5) and their receptors (APOBR). Down-regulation of this miRNA in the adipose tissue of obese individuals clarifies one of the mechanisms of obesity-related low-grade inflammation and lipid disturbances [24].

Using the microarray technique, Capobianco et al. identified several miRNAs differentially expressed in VAT of obese and normal-weight individuals [25]. However in the NGS analysis, the only miRNA significantly up-regulated in VAT-O compared to VAT-N was hsa-miR-424-3p that acts as a regulator of the nuclear factor IA (NFIA), a transcription factor required for proper adipocyte differentiation and lipid droplet formation. Therefore, its down-regulation may contribute to adipose tissue dysfunction [26].

Loss of weight was associated with significant changes in the adipose tissue miRNA profile. These changes concerned, among others, the balance between miRNAs involved in the regulation of adipogenesis. Notably, the expression of only one adipogenesis-related miRNA, hsa-miR-146b-3p, that was higher in SAT-O compared to SAT-N, decreased in SAT-PO to the level observed in SAT-N. In addition, we observed a decreased expression of several proadipogenic miRNAs, namely hsa-miR-15a-5p, hsa-miR-107, hsa-miR-143-5p, hsa-miR-194-5p and hsa-miR-210, and an increased expression of the antiadipogenic hsa-miR-196a-5p [13] in SAT-PO vs. SAT-O, but their expression did not return to the levels observed in SAT-N, suggesting that they may represent permanent miRNome changes induced by obesity. Surprisingly, the levels of hsa-miR-18a-3p and hsa-miR-130b-3p, described previously as antiadipogenic, were decreased in SAT-PO [13,27]. In the study by Ortega et al. the expression of hsa-miR-130b was also decreased in adipocytes isolated from the SAT of obese subjects after weight loss; however, as mentioned above, the assay applied by this author did not distinguish between the two hsa-miR-130b isoforms [8]. Compared to SAT-O, SAT-PO was also characterized by a different expression of several miRNAs involved in the regulation of the immune response and ontological analyses pointed to an underrepresentation of inflammatory pathways in post-bariatric SAT. Among other miRNAs with a lower expression in SAT-PO were those crucial for lipid metabolism, such as hsa-miR-144-3p involved in the decrease of HDL formation [28], for insulin signaling such as hsa-miR-96-5p that represses expression of the gene encoding insulin receptor INSR [29], for ageing such as hsa-miR-141-3p that accelerates the ageing of human mesenchymal cells [30], and for those promoting the development of cardiovascular and neurodegenerative diseases, such as hsa-miR-3615 and hsa-miR-15b-5p [31,32]. Therefore, the decreased expression of these genes indicates one of the mechanisms by which loss of weight lowers the risk of obesity-associated complications. Loss of weight was also associated with a lower expression of several miRNAs involved in the regulation of oncogenesis, while ontological analyses suggested overrepresentation of pathways involved in apoptosis signaling and p53-action in SAT-PO. Notably, what also distinguished SAT-PO from SAT-N, was the overrepresentation of signaling transmitted via cholecystokinine receptors (CCKR) which increase sympathetic nerve activity in brown adipose tissue, enhancing its termogenic activity [33]. To sum up, the miRNome of SAT after loss of weight, despite positive changes compared to the SAT of the obese, still differed from that of normal-weight subjects, suggesting that even after the normalization of body mass index (BMI), a period of obesity leaves a long-lasting imprint on the metabolism of adipose tissue regarding control of functions such as adipogenesis, lipid metabolism, the immune response, insulin signaling, atherosclerosis, ageing, cardiovascular diseases, neurodegenerative disorders and oncogenesis [13,28-32]. 
Finally, we found that while in normal-weight individuals there was no significant difference in miRNA levels between visceral and subcutaneous adipose tissue, obesity was associated with pronounced differences in the miRNomes of these two depots (Table 2). Consequently, ontological analyses identified several molecular pathways differentially regulated in the VAT and SAT of obese subjects. VAT was characterized by the overrepresentation of pathways associated with inflammation and interleukin signaling. Interpretation of this finding is somewhat difficult since some gene expression analyses pointed to increased pro-inflammatory activity of VAT [1], while other authors obtained the opposite results $[19,34]$. One of the pathways underrepresented in VAT was that related to presenilin action. Activation of this pathway was detected in transgenic mice genetically predisposed to the development of dementia, in which bodyweight gain and hyperglycemia resulted in increased serum $\beta$-amyloid levels [35] In turn, a distinct regulation of pathways associated with glycolysis and WNT (wingless-type MMTV integration site family members) signaling in VAT-O vs. SAT-O, may reflect different potentials of these depots to accumulate excess fat in obese subjects [36]. WNTs are glycoproteins involved in control of cell proliferation and survival and in the context of adipose tissue, WNT canonical signaling pathways were found to restrain differentiation of mesenchymal stem cells towards adipocytes [37].

In summary, a comprehensive, NGS-based miRNome analysis revealed significant differences in miRNA levels between different adipose tissue depots originating from obese and normal-weight individuals, and characterized changes in miRNA profile resulting from weight loss. Bioinformatics tools applied to detect genes and pathways affected by changes in miRNA levels identified pathways involved in the regulation of inflammation, cytokine signaling, adrenergic receptors action and apoptosis. These findings constitute the basis for subsequent functional studies on the pathogenesis of obesity and related complications.

\section{Materials and Methods}

\subsection{Study Groups}

Pairs of visceral (VAT) and subcutaneous (SAT) adipose tissues were obtained from 58 obese patients $\left(\mathrm{O}\right.$, body mass index $\left.(\mathrm{BMI})>40 \mathrm{~kg} / \mathrm{m}^{2}\right)$ during bariatric surgery, as described previously [38]. Fifty-five control tissues were collected from normal-weight individuals (N, BMI $20-24.9 \mathrm{~kg} / \mathrm{m}^{2}$ ) undergoing elective cholecystectomy (24 samples of VAT and 24 samples of SAT) or operated for inguinal hernia (seven samples of SAT). Nineteen additional samples of SAT were collected from formerly obese subjects about two years after surgery-induced weight loss (PO, BMI $24.3-29.5 \mathrm{~kg} / \mathrm{m}^{2}$ ). VAT samples from the PO subjects were unavailable since post-bariatric surgery regards only skin fold removal and is not associated with abdominal cavity opening. Basic clinical characteristics of study participants are summarized in Table S2. The project was approved by the Bioethics Committee of the Medical University of Warsaw, and written informed consent for participation in this study was obtained from all participants.

\section{2. miRNA Expression Analysis by Next Generation Sequencing}

After collection, all adipose tissue samples were immediately frozen at $-80^{\circ} \mathrm{C}$, homogenized in liquid nitrogen and stored again at $-80^{\circ} \mathrm{C}$. The previously described methods [20] were used for total RNA isolation. RNA integrity was assessed using an Agilent 2100 Bioanalyzer (Agilent Technology, Santa Clara, CA, USA). For NGS, only samples with the highest quality and integrity (RNA integrity number (RIN) $>8$ ) were selected, so this analysis was performed on 44 tissues: 10 pairs of VAT and SAT from the obese study participants $(\mathrm{O}$, eight women, two men), seven pairs of VAT and SAT from normal-weight subjects ( $\mathrm{N}$, six women, one man) and 10 SAT samples from individuals after surgery-induced weight loss ( $\mathrm{PO}$, eight women, two men).

A small fraction of RNA was isolated, sequenced and analyzed as described previously [10]. Data obtained for each sample were normalized using the RPM normalization according to the formula: 
$\mathrm{RPM}=\left(\mathrm{N}_{\text {ref }} / \mathrm{N}_{\text {all }}\right) \times 10^{6}$, where $\mathrm{N}_{\text {ref }}$ is the number of reads mapped to the miRNA reference and $\mathrm{N}_{\text {all }}$ is the total number of reads mapped to the sample.

\subsection{Validation of miRNA Expression by Real-Time PCR}

The direction of the NGS-detected obesity-associated expression changes was established for 19 miRNAs. For this analysis, we selected 16 miRNAs for which NGS revealed the most significant differences in their expression between the investigated tissues (hsa-miR-22-3p, hsa-miR-96-5p, hsa-miR-125a-5p, hsa-miR-125b-5p, hsa-miR-146b-3p, hsa-miR-183-5p, hsa-miR-193a-3p, hsa-miR-193b-5p, hsa-miR-194-5p, hsa-miR-196a-5p, hsa-miR-205-5p, hsa-miR-223-3p, hsa-miR-424-3p, hsa-miR-450b-5p, hsa-miR-486-5p and hsa-miR-1260b). We also assessed levels of three miRNAs with the most stable expression in NGS (hsa-miR-214-3p, hsa-miR-342-3p and hsa-miR-374a-5p). The analysis of miRNAs expression was performed with the miRCURY LNA ${ }^{\mathrm{TM}}$ Universal RT microRNA PCR system (Exiqon, Vedbaek, Denmark) according to the manufacturer's protocol, as described previously [38]. Reactions were performed in triplicate using RNA from 197 adipose tissues. The results were normalized against the miR-103a-3p expression, the recommended control miRNA for the adipose tissue [2]. The RNA spike-ins provided by the manufacturer, were used to control the quality of RNA isolation and of cDNA synthesis, and as an inter-plate calibrator.

\subsection{Identification of miRNA Target Genes and Their Molecular Pathways}

The MirWalk (http://zmf.umm.uni-heidelberg.de/apps/zmf/mirwalk2) and MirTarBase (http:/ / mirtarbase.mbc.nctu.edu.tw) programs were used to identify target genes regulated by miRNAs with differential expression [39-42].

Putative target genes were first identified by miRWalk which utilizes a restrictive algorithm to predict mRNA-miRNA interaction sites by comparing the complete nuclear genes and mitochondrial DNA sequences with miRNA 7-nucleotide seed region and by calculating the probability distribution of random matches of a subsequence from the $5^{\prime}$ end of miRNA sequence, using the Poisson distribution. Next, the program compared the obtained results with results from other popular prediction programs: DIANA-microT, miRanda, miRDB, PicTar, Probability of Interaction by Target Accessibility (PITA), RNA22, RNAhybrid and TargetScan/TargetScanS. This analysis was complemented by data from the Advanced Search tool from MirTarBase containing interaction sites that had been verified by functional tests (reporter assay, immunoblot, qPCR, microarray, protein stable isotope labeling with amino acids in cell culture (pSILAC), cross-linking immunoprecipitation followed by sequencing (CLIP-seq)).

A rough estimation of the direction of target gene expression changes was obtained as follows: when miRNA had a lower expression in a given tissue than in another tissue with which it was compared, we assigned the value of +1 to the expression of its target gene and opposite, when miRNA expression was higher than in the other tissue, we assigned the value of -1 . For each gene, the respective values were summarized. When the sum had a positive value, we considered the expression of the gene to be increased, while a negative value indicated gene suppression. Results of this estimation were verified for 10 genes (enlisted in the Results section) by real-time PCR as described previously, with specific primers enlisted in Table S3 [38].

Subsequently, target genes were analyzed with the PANTHER (Protein ANalysis THrough Evolutionary Relationships) Classification System v. 11.0 (Paul Thomas Lab, Los Angeles, CA, USA) combining gene functions, ontology and pathways [43]. Based on this analysis, molecular pathways that were regulated by miRNAs with differential expression were identified. Differences in the regulation of pathways were presented as fold enrichment. Fold enrichment values $>1$ corresponded to the overrepresentation (increased regulation) of the particular pathway, while values $<1$ to its underrepresentation (decreased regulation) as compared to control tissue from a given pair of tissues. 


\subsection{Statistical Analysis}

NGS analysis and selection of differentially expressed miRNAs were performed using the $\mathrm{R} /$ Bioconductor (Roswell Park Cancer Institute, Buffalo, NY, USA) software with paired Welch $t$-test. False discovery rate (FDR) was used to assess the multiple testing errors.

Analysis regarding RT-PCR data on miRNA and mRNA expression was performed with the Statistica Version 7.0 software package (StatSoft, Tulsa, OK, USA) using Student's t or Mann-Whitney's $U$ test when appropriate. Assessment of normality of the distribution was performed with the Shapiro-Wilk test.

To identify molecular pathways, the sets of genes were analyzed using the binomial overrepresentation test with Bonferroni correction from the Statistical Enrichment Test tool included in the PANTHER Classification System program v. 11.0 (Paul Thomas Lab, Los Angeles, CA, USA).

Supplementary Materials: Supplementary materials can be found at www.mdpi.com/1422-0067/19/1/66/s1.

Acknowledgments: This work was supported by the National Science Centre Poland grant 2012/05/B/NZ5/01536.

Author Contributions: Alina Kuryłowicz conceived of the project and carried out experiments, analyzed data and wrote the paper, Zofia Wicik, Magdalena Owczarz, Marta Izabela Jonas, Marta Kotlarek and Michał Świerniak carried out experiments and analyzed data, Wojciech Lisik, Maurycy Jonas and Bartłomiej Noszczyk collected tissues and clinical data, Monika Puzianowska-Kuznicka participated in project development, analyzed the data and wrote the paper. All authors approved the final version of the manuscript.

Conflicts of Interest: The authors declare no conflict of interests.

\section{References}

1. Samaras, K.; Botelho, N.K.; Chisholm, D.J.; Lord, R.V. Subcutaneous and visceral adipose tissue gene expression of serum adipokines that predict type 2 diabetes. Obesity 2010, 18, 884-889. [CrossRef] [PubMed]

2. Neville, M.J.; Collins, J.M.; Gloyn, A.L.; McCarthy, M.I.; Karpe, F. Comprehensive human adipose tissue mRNA and microRNA endogenous control selection for quantitative real-time-PCR normalization. Obesity 2011, 19, 888-892. [CrossRef] [PubMed]

3. Gauthier, M.S.; Pérusse, J.R.; Lavoie, M.È.; Sladek, R.; Madiraju, S.R.; Ruderman, N.B.; Coulombe, B.; Prentki, M.; Rabasa-Lhoret, R. Increased subcutaneous adipose tissue expression of genes involved in glycerolipid-fatty acid cycling in obese insulin-resistant versus -sensitive individuals. J. Clin. Endocrinol. Metab. 2014, 99, E2518-E2528. [CrossRef] [PubMed]

4. Kirby, T.J.; Walton, R.G.; Finlin, B.; Zhu, B.; Unal, R.; Rasouli, N.; Peterson, C.A.; Kern, P.A. Integrative mRNA-microRNA analyses reveal novel interactions related to insulin sensitivity in human adipose tissue. Physiol. Genom. 2016, 48, 145-153. [CrossRef] [PubMed]

5. Xie, H.; Lim, B.; Lodish, H.F. MicroRNAs induced during adipogenesis that accelerate fat cell development are downregulated in obesity. Diabetes 2009, 58, 1050-1057. [CrossRef] [PubMed]

6. Oger, F.; Gheeraert, C.; Mogilenko, D.; Benomar, Y.; Molendi-Coste, O.; Bouchaert, E.; Caron, S.; Dombrowicz, D.; Pattou, F.; Duez, H.; et al. Cell-specific dysregulation of microRNA expression in obese white adipose tissue. J. Clin. Endocrinol. Metab. 2014, 99, 2821-2833. [CrossRef] [PubMed]

7. Ortega, F.J.; Moreno-Navarrete, J.M.; Pardo, G.; Sabater, M.; Hummel, M.; Ferrer, A.; Rodriguez-Hermosa, J.I.; Ruiz, B.; Ricart, W.; Peral, B.; et al. MiRNA expression profile of human subcutaneous adipose and during adipocyte differentiation. PLOS ONE 2010, 5, e9022. [CrossRef] [PubMed]

8. Ortega, F.J.; Mercader, J.M.; Moreno-Navarrete, J.M.; Nonell, L.; Puigdecanet, E.; Rodriquez-Hermosa, J.I.; Rovira, O.; Xifra, G.; Guerra, E.; Moreno, M.; et al. Surgery-Induced Weight Loss Is Associated With the Downregulation of Genes Targeted by MicroRNAs in Adipose Tissue. J. Clin. Endocrinol. Metab. 2015, 100, E1467-E1476. [CrossRef] [PubMed]

9. Klöting, N.; Berthold, S.; Kovacs, P.; Schön, M.R.; Fasshauer, M.; Ruschke, K.; Stumvoll, M.; Blüher, M. MicroRNA expression in human omental and subcutaneous adipose tissue. PLoS ONE 2009, 4, e4699. [CrossRef] [PubMed] 
10. Swierniak, M.; Wojcicka, A.; Czetwertynska, M.; Stachlewska, E.; Maciag, M.; Wiechno, W.; Gornicka, B.; Bogdanska, M.; Koperski, L.; de la Chapelle, A.; et al. In-depth characterization of the microRNA transcriptome in normal thyroid and papillary thyroid carcinoma. J. Clin. Endocrinol. Metab. 2013, 98, E1401-E1409. [CrossRef] [PubMed]

11. Wojcicka, A.; Swierniak, M.; Kornasiewicz, O.; Gierlikowski, W.; Maciag, M.; Kolanowska, M.; Kotlarek, M.; Gornicka, B.; Koperski, L.; Niewinski, G.; et al. Next generation sequencing reveals microRNA isoforms in liver cirrhosis and hepatocellular carcinoma. Int. J. Biochem. Cell Biol. 2014, 53, 208-217. [CrossRef] [PubMed]

12. Arner, P.; Kulyté, A. MicroRNA regulatory networks in human adipose tissue and obesity. Nat. Rev. Endocrinol. 2015, 11, 276-288. [CrossRef] [PubMed]

13. Qin, L.; Chen, Y.; Niu, Y.; Chen, W.; Wang, Q.; Xiao, S.; Li, A.; Xie, Y.; Li, J.; Zhao, X.; et al. A deep investigation into the adipogenesis mechanism: Profile of microRNAs regulating adipogenesis by modulating the canonical Wnt/beta-catenin signaling pathway. BMC Genom. 2010, 11, 320. [CrossRef] [PubMed]

14. Nardelli, C.; Iaffaldano, L.; Pilone, V.; Labruna, G.; Ferrigno, M.; Carlomagno, N.; Dodaro, C.A.; Forestieri, P.; Buono, P.; Salvatore, F.; et al. Changes in the MicroRNA Profile Observed in the Subcutaneous Adipose Tissue of Obese Patients after Laparoscopic Adjustable Gastric Banding. J. Obes. 2017, 2017, 6754734. [CrossRef] [PubMed]

15. Keller, P.; Gburcik, V.; Petrovic, N.; Gallagher, I.J.; Nedergaard, J.; Cannon, B.; Timmons, J.A. Gene-chip studies of adipogenesis-regulated microRNAs in mouse primary adipocytes and human obesity. BMC Endocr. Disord. 2011, 11, 7. [CrossRef] [PubMed]

16. Hulsmans, M.; Van Dooren, E.; Mathieu, C.; Holvoet, P. Decrease of miR-146b-5p in monocytes during obesity is associated with loss of the anti-inflammatory but not insulin signaling action of adiponectin. PLoS ONE 2012, 7, e32794. [CrossRef] [PubMed]

17. Ogando, J.; Tardáguila, M.; Díaz-Alderete, A.; Usategui, A.; Miranda-Ramos, V.; Martínez-Herrera, D.J.; de la Fuente, L.; García-León, M.J.; Moreno, M.C.; Escudero, S.; et al. Notch-regulated miR-223 targets the aryl hydrocarbon receptor pathway and increases cytokine production in macrophages from rheumatoid arthritis patients. Sci. Rep. 2016, 6, 20223. [CrossRef] [PubMed]

18. Greenberg, A.S.; Obin, M.S. Obesity and the role of adipose tissue in inflammation and metabolism. Am. J. Clin. Nutr. 2006, 83, 461S-465S. [PubMed]

19. Jonas, M.I.; Kurylowicz, A.; Bartoszewicz, Z.; Lisik, W.; Jonas, M.; Wierzbicki, Z.; Chmura, A.; Pruszczyk, P.; Puzianowska-Kuznicka, M. Interleukins 6 and 15 Levels Are Higher in Subcutaneous Adipose Tissue, but Obesity Is Associated with Their Increased Content in Visceral Fat Depots. Int. J. Mol. Sci. 2015, 16, 25817-25830. [CrossRef] [PubMed]

20. Schinzari, F.; Tesauro, M.; Cardillo, C. Endothelial and perivascular adipose tissue abnormalities in obesity-related vascular dysfunction: Novel targets for treatment. J. Cardiovasc. Pharmacol. 2017, 69, 360-368. [CrossRef] [PubMed]

21. Alkhouri, N.; Gornicka, A.; Berk, M.P.; Thapaliya, S.; Dixon, L.J.; Kashyap, S.; Schauer, P.R.; Feldstein, A.E. Adipocyte apoptosis, a link between obesity, insulin resistance, and hepatic steatosis. J. Biol. Chem. 2010, 285, 3428-3438. [CrossRef] [PubMed]

22. Vergoni, B.; Cornejo, P.J.; Gilleron, J.; Djedaini, M.; Ceppo, F.; Jacquel, A.; Bouget, G.; Ginet, C.; Gonzalez, T.; Maillet, J.; et al. DNA Damage and the Activation of the p53 Pathway Mediate Alterations in Metabolic and Secretory Functions of Adipocytes. Diabetes 2016, 65, 3062-3074. [CrossRef] [PubMed]

23. Kurylowicz, A.; Jonas, M.; Lisik, W.; Jonas, M.; Wicik, Z.A.; Wierzbicki, Z.; Chmura, A.; Puzianowska-Kuznicka, M. Obesity is associated with a decrease in expression but not with the hypermethylation of thermogenesis-related genes in adipose tissues. J. Transl. Med. 2015, 13, 31. [CrossRef] [PubMed]

24. Lopategi, A.; López-Vicario, C.; Alcaraz-Quiles, J.; García-Alonso, V.; Rius, B.; Titos, E.; Clària, J. Role of bioactive lipid mediators in obese adipose tissue inflammation and endocrine dysfunction. Mol. Cell. Endocrinol. 2016, 419, 44-59. [CrossRef] [PubMed]

25. Capobianco, V.; Nardelli, C.; Ferrigno, M.; Iaffaldano, L.; Pilone, V.; Forestieri, P.; Zambrano, N.; Sacchetti, L. miRNA and protein expression profiles of visceral adipose tissue reveal miR-141/YWHAG and miR-520e/RAB11A as two potential miRNA/protein target pairs associated with severe obesity. J. Proteome Res. 2012, 11, 3358-3369. [CrossRef] [PubMed] 
26. Hu, Y.W.; Zhao, J.Y.; Li, S.F.; Huang, J.L.; Qiu, Y.R.; Ma, X.; Wu, S.G.; Chen, Z.P.; Hu, Y.R.; Yang, J.Y.; et al. RP5-833A20.1/miR-382-5p/NFIA-dependent signal transduction pathway contributes to the regulation of cholesterol homeostasis and inflammatory reaction. Arterioscler. Thromb. Vasc. Biol. 2015, 35, 87-101. [CrossRef] [PubMed]

27. Shi, C.; Huang, F.; Gu, X.; Zhang, M.; Wen, J.; Wang, X.; You, L.; Cui, X.; Ji, C.; Guo, X. Adipogenic miRNA and meta-signature miRNAs involved in human adipocyte differentiation and obesity. Oncotarget 2016, 7, 40830-40845. [CrossRef] [PubMed]

28. De Aguiar Vallim, T.Q.; Tarling, E.J.; Kim, T.; Civelek, M.; Baldán, Á.; Esau, C.; Edwards, P.A. MicroRNA-144 regulates hepatic ATP binding cassette transporter A1 and plasma high-density lipoprotein after activation of the nuclear receptor farnesoid X receptor. Circ. Res. 2013, 112, 1602-1612. [CrossRef] [PubMed]

29. Yang, W.M.; Min, K.H.; Lee, W. Induction of miR-96 by Dietary Saturated Fatty Acids Exacerbates Hepatic Insulin Resistance through the Suppression of INSR and IRS-1. PLoS ONE 2016, 11, e0169039. [CrossRef] [PubMed]

30. Yu, K.R.; Lee, S.; Jung, J.W.; Hong, I.S.; Kim, H.S.; Seo, Y.; Shin, T.H.; Kang, K.S. MicroRNA-141-3p plays a role in human mesenchymal stem cell aging by directly targeting ZMPSTE24. J. Cell Sci. 2013, 126, 5422-5431. [CrossRef] [PubMed]

31. Liang, J.; Bai, S.; Su, L.; Li, C.; Wu, J.; Xia, Z.; Xu, D. A subset of circulating microRNAs is expressed differently in patients with myocardial infarction. Mol. Med. Rep. 2015, 12, 243-247. [CrossRef] [PubMed]

32. Kumar, P.; Dezso, Z.; MacKenzie, C.; Oestreicher, J.; Agoulnik, S.; Byrne, M.; Bernier, F.; Yanagimachi, M.; Aoshima, K.; Oda, Y. Circulating miRNA biomarkers for Alzheimer's disease. PLoS ONE 2013, 8, e69807. [CrossRef] [PubMed]

33. Madden, C.J.; Tupone, D.; Cano, G.; Morrison, S.F. $\alpha 2$ Adrenergic receptor-mediated inhibition of thermogenesis. J. Neurosci. 2013, 33, 2017-2028. [CrossRef] [PubMed]

34. Spoto, B.; Di Betta, E.; Mattace-Raso, F.; Sijbrands, E.; Vilardi, A.; Parlongo, R.M.; Pizzini, P.; Pisano, A.; Vermi, W.; Testa, A.; et al. Pro- and anti-inflammatory cytokine gene expression in subcutaneous and visceral fat in severe obesity. Nutr. Metab. Cardiovasc. Dis. 2014, 24, 1137-1143. [CrossRef] [PubMed]

35. Shie, F.S.; Shiao, Y.J.; Yeh, C.W.; Lin, C.H.; Tzeng, T.T.; Hsu, H.C.; Huang, F.L.; Tsay, H.J.; Liu, H.K. Obesity and Hepatic Steatosis Are Associated with Elevated Serum Amyloid Beta in Metabolically Stressed APPswe/PS1dE9 Mice. PLoS ONE 2015, 10, e0134531. [CrossRef] [PubMed]

36. Young, P.; Cawthorne, M.A.; Levy, A.L.; Wilson, K. Reduced maximum capacity of glycolysis in brown adipose tissue of genetically obese, diabetic $(\mathrm{db} / \mathrm{db})$ mice and its restoration following treatment with a thermogenic beta-adrenoceptor agonist. FEBS Lett. 1984, 176, 16-20. [CrossRef]

37. Christodoulides, C.; Lagathu, C.; Sethi, J.K.; Vidal-Puig, A. Adipogenesis and WNT signalling. Trends Endocrinol. Metab. 2009, 20, 16-24. [CrossRef] [PubMed]

38. Kurylowicz, A.; Owczarz, M.; Polosak, J.; Jonas, M.I.; Lisik, W.; Jonas, M.; Chmura, A.; Puzianowska-Kuznicka, M. SIRT1 and SIRT7 expression in adipose tissues of obese and normal-weight individuals is regulated by microRNAs but not by methylation status. Int. J. Obes. 2016, 40, 1635-1642. [CrossRef] [PubMed]

39. miRWalk2.0: A Comprehensive Atlas of Predicted and Validated miRNA-Target Interactions. Available online: http://zmf.umm.uni-heidelberg.de/apps/zmf/mirwalk2/ (accessed on 18 October 2017).

40. Dweep, H.; Gretz, N.; Sticht, C. miRWalk database for miRNA-target interactions. Methods Mol. Biol. 2014, 1182, 289-305. [CrossRef] [PubMed]

41. miRTarBase: The Experimentally Validated microRNA-Target Interactions Database. Available online: http:/ / mirtarbase.mbc.nctu.edu.tw (accessed on 18 October 2017).

42. Chou, C.H.; Shrestha, S.; Yang, C.D.; Chang, N.W.; Lin, Y.L.; Liao, K.W.; Huang, W.C.; Sun, T.H.; Tu, S.J.; Lee, W.H.; et al. miRTarBase update 2018: A resource for experimentally validated microRNA-target interactions. Nucleic Acids Res. 2017. [CrossRef] [PubMed]

43. Mi, H.; Muruganujan, A.; Casagrande, J.T.; Thomas, P.D. Large-scale gene function analysis with the PANTHER classification system. Nat. Protoc. 2013, 8, 1551-1566. [CrossRef] [PubMed]

(C) 2017 by the authors. Licensee MDPI, Basel, Switzerland. This article is an open access article distributed under the terms and conditions of the Creative Commons Attribution (CC BY) license (http:/ / creativecommons.org/licenses/by/4.0/). 